\title{
Acute Left Ventricular Outflow Tract Obstruction in Non- Mitral Cardiovascular Surgery: A Case Series Analysis
}

\author{
Krichevskiy LA ${ }^{1}$, Rybakov VY1 ${ }^{1}$, Guseva OG $^{2}$ and Guseva N V ${ }^{1}$ \\ ${ }_{1}^{1}$ Department of Cardiac Anesthesia and Intensive Care, S.S.Yudin City Hospital, Russia \\ ${ }^{2}$ Department of Cardiac Anesthesia and Intensive Care, O.M.Filatov City Hospital, Russia \\ *Corresponding author: Lev Krichevskiy, S.S.Yudin City Hospital, Department of \\ Cardiac Anesthesia and Intensive Care, Moscow, Russia, Tel: 9035679427; Email: \\ levkrich72@gmail.com
}

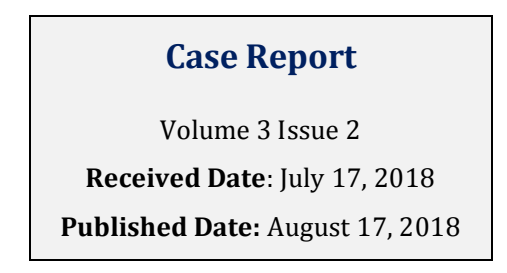

\section{Abstract}

Objective: We aimed to analyze the clinical signs of left ventricular outflow tract obstruction and its management in the perioperative period of major non-mitral cardiovascular procedures. Design. Case series analysis. Methods and Results Thirteen (10 males, 3 females) patients aged 64 (56; 74) y.o. with acutely emerged left ventricular outflow tract obstruction during/after non-mitral cardiovascular procedure between May 2006 and May 2018 were included.

Methods: The procedures were as follows: coronary artery bypass grafting $-n=11$, aortic valve replacement $-n=1$, abdominal aortic membrane resection (aortic dissection DeBakey type I, acute legs ischemia) - n=1. Left ventricular outflow tract obstruction with systolic anterior motion of anterior leaflet of mitral valve was detected in $0.9 \%$ of the total number of perioperative echocardiography examinations. Three variants of its clinical course were described: (1) intracardiac and systemic hemodynamics recovery with a specific therapy (most cases); (2) full resistance to therapy with sustainable systolic anterior motion persistence; (3) termination of systolic anterior motion as a result of the therapy, but the paradoxical persistence of low cardiac output syndrome.

Conclusion: Practitioners' vigilance and Echocardiographic monitoring are needed for early detection of acute left ventricular outflow tract obstruction. Its development can be a marker of the extremely hard concentric left ventricular hypertrophy as a cause of the low cardiac output syndrome. Key Words Systolic anterior motion, left ventricular outflow tract obstruction, left ventricular hypertrophy, cardiac surgery.

Keywords: Cardiovascular Surgery; Hypertrophic cardiomyopathy; Echocardiographic monitoring; ventricular outflow

Abbreviations: LVOTO: Left ventricular outflow tract obstruction; AML: Anterior mitral leaflet; MVR: Mitral valve repair; AVR: Aortic valve replacement; LVEF: Left ventricular ejection fraction; PAC: Pulmonary artery catheter; CABG: Coronary artery bypass grafting; LVH: Left ventricular hypertrophy. 


\section{Anaesthesia \& Critical Care Medicine Journal}

\section{Introduction}

Left ventricular outflow tract obstruction (LVOTO) due to systolic anterior motion (SAM) of the anterior mitral leaflet (AML), which typically occurs in patients with hypertrophic cardiomyopathy [1,2], is well known as a complication of mitral valve repair (MVR) [1,3-7]. During non-mitral cardiovascular surgery, such as coronary artery bypass grafting (CABG) or aortic valve replacement (AVR), LVOTO has been described almost exclusively in detached small studies $[8,9]$. Some case reports also described acute LVOTO during liver transplant and other major surgical procedures for which transesophageal echocardiography (TEE) is used routinely [10-14]. The predictors of this disturbance are left ventricular hypertrophy (LVH), hyper dynamic left ventricular ejection fraction (LVEF) [15], Hypovolemia, arterial hypotension, and inotropic therapy $[1,16]$. These factors can promote the Venturi effect with the development of suction force in LVOT leading to SAM. The size of AML and reduced mitro-aortic angle are important contributing factors in MVR cases [16-18]. Conventional hemodynamic monitoring, including pulmonary artery catheter (PAC) is not fully potent to diagnose LVOTO. In fact, the effectiveness of diagnostics and the intensity of the registration of SAM are dependent on the use of echocardiography for routine monitoring. The detection of SAM has been speculated to be a criterion of sufficient educational and qualifying levels of the anesthesiological team [19]. We aimed to describe and analyze the clinical and Echocardiographic signs of LVOTO in the perioperative period of major cardiovascular procedures (Figure 1).

\section{Methods}

All consecutive patients with acute LVOTO during or after major cardiovascular procedures from September 2008 to May 2018, except MV surgery, were included. Echocardiography. In all cases, the diagnosis of LVOTO/SAM was identified by multiplane TEE using 2D, color Doppler, pulse wave (PW), and continuous wave (CW) Doppler modes. The criteria of LVOTO were typical abnormal AML systolic motion, peak gradient in LVOT $>40 \mathrm{mmHg}$, mitral regurgitation (MR) II-IV, and asymmetric LVH with interventricular septum (IVS) bulging [1]. A typical echocardiogram is shown in the figure 1 . Standard hemodynamic monitoring. All patients were monitored with invasive arterial pressure (AP), PAC (before and/or just after LVOTO diagnostics) to control pulmonary arterial pressure (PAP), pulmonary arterial wedge pressure (PAWP), and cardiac index (CI). Standard LVOTO therapy generally involves inotropic support cancellation, beta-blockers, fluid loading, and arterial hypertension induction by phenylephrine.

Data collection and statistical analysis were performed using Microsoft Excel 2010 for Windows 8. Statistical data were reported as median (interquartile range, IQR). The Mann-Whitney U-test was used for data comparison. Pvalues $<0.05$ were considered significant.

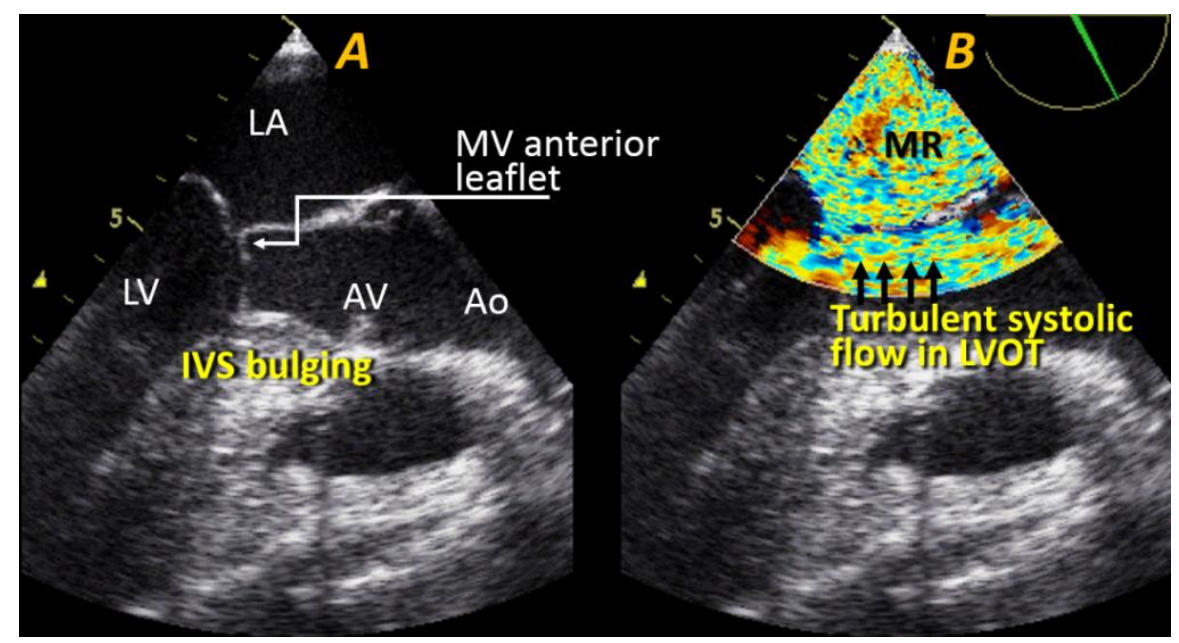

Figure1: Transesophageal echocardiography, midesophageal long axis views (ME LAX). Systolic anterior motion of anterior leaflet of mitral valve just after on-pump coronary artery bypasses grafting. A - 2D mode; B - Color Doppler mode

Abbreviations: LA - Left atrium; LV - Left ventricle, AV - Aortic valve, MV - Mitral valve; Ao - Aorta; IVS Interventricular septum; MR - Mitral regurgitation; LVOT - Left ventricular outflow tract. 


\section{Anaesthesia \& Critical Care Medicine Journal}

\section{Results}

Thirteen patients (10 men, 3 women) aged 64 years (IQR 56;74) with acute LVOTO during/after cardiovascular surgical procedures were identified. These surgical procedures were on-pump coronary artery bypass grafting (CABG, $n=11$ ), aortic valve replacement (AVR) due to aortic stenosis (AS, $n=1$ ), abdominal aortic membrane resection (aortic dissection DeBakey type I with acute leg ischemia, $n=1$ ).

Asymmetric LVH was diagnosed only preoperatively in five $(38.5 \%)$ patients. Before surgery, LVEF was $>50 \%$ in all patients (Table 1). The significant increase in LVEF and IVST were recorded during perioperative LVOTO diagnostics. Acute MR II-IV and PG in LVOTO $>40 \mathrm{mmHg}$ were presented in all patients.

\begin{tabular}{|c|c|c|c|}
\hline Parameters & $\begin{array}{c}\text { Preoperatively } \\
\text { (TTE) }\end{array}$ & $\begin{array}{c}\text { LVOTO/SAM } \\
\text { detection (TEE) }\end{array}$ & P \\
\hline LVEF, \% & $56(55 ; 64)$ & $78(73 ; 84)$ & $<0.01$ \\
\hline LVIWT, mm & $11(10 ; 14)$ & $12(11 ; 13)$ & $>0.05$ \\
\hline IVST, mm & $11(10 ; 14)$ & $17(14 ; 22)$ & $<0.01$ \\
\hline $\begin{array}{c}\text { PG LVOT, } \\
\text { mmHg }\end{array}$ & None & $77(65 ; 84)$ & $<0.01$ \\
\hline MR, $^{\circ}$ & None & $3(3 ; 4)$ & $<0.01$ \\
\hline
\end{tabular}

Table 1: Echocardiographic data.

Abbreviations: TTE- transthoracic echocardiography; TEE-transesophageal echocardiography; LVEF-left ventricular ejection fraction; LVIWT- left ventricular inferior wall thickness; IVST- interventricular septum thickness; PG LVOT-peak gradient in the left ventricular outflow tract; MR-mitral regurgitation

NOTE. Data are presented as median (interquartile range).

Cancellation of inotropic support, $15 \mathrm{ml}^{*} \mathrm{~kg}^{-1}$ (IQR $11 ; 22$ ) of fluid rapid infusion, and $0.08 \mathrm{mg}^{*} \mathrm{~kg}^{-1}$ (IQR 0.06 ; $0.09)$ of metoprolol were used $(n=13)$. In four cases, phenylephrine-induced arterial hypertension (systolic arterial pressure [AP] up to $160 \mathrm{mmHg}$ ) was additionally required. In one patient, the atrioventricular (AV) pacing with short $(70 \mathrm{~ms})$ delay was used because of the ineffectiveness of other interventions. LVOTO was terminated in 12 patients, and $\mathrm{CI}$ increased to $>2.5$ $\mathrm{L}^{*} \mathrm{~min}^{-1 *} \mathrm{~m}^{-2}$ in 10 patients. This therapeutic algorithm was unsuccessful in two patients described below.

CABG (4 grafts) was performed with cardiopulmonary bypass (CPB) in an 84-year-old woman. Moderate LVH was described preoperatively: the IVS thickness (IVST), LV inferior wall thickness (LVIWT), and LVEF were $14 \mathrm{~mm}, 12 \mathrm{~mm}$, and 64\%, respectively. Lactic acidosis and dopamine requirements were the indications for PAC insertion $6 \mathrm{~h}$ postoperatively. LCOS (CI, 1.7 $\mathrm{L}^{*} \mathrm{~min}^{-1 *} \mathrm{~m}^{-2}$ ) was found, perioperative myocardial infarction was preliminarily diagnosed, and intra-aortic balloon pumping (IABP) was started. Subsequently, urgent TEE was performed, and typical LVOTO was presented: abnormal AML movement; IVST, $24 \mathrm{~mm}$; peak pressure gradient in LVOT, $97 \mathrm{mmHg}$; LVEF, 82\%; and total mitral regurgitation. Dopamine and IABP were discontinued, fluid loading $\left(17 \mathrm{ml}^{*} \mathrm{~kg}^{-1}\right)$, bolus and continuous infusion of metoprolol were used, arterial hypertension up to systolic AP of $160 \mathrm{mmHg}$ was maintained using phenylephrine. Unfortunately, despite this intensive treatment, SAM with severe LCOS (CI, $<2.0$ $\mathrm{L}^{*} \mathrm{~min}^{-1}{ }^{*} \mathrm{~m}^{-1}$, lactate level $>8 \mathrm{mmol}^{*} \mathrm{~L}^{-1}$ ) persisted during the entire postoperative period ( 6 days) until the patient died.

CABG (three grafts) with CPB was performed in a 64year-old man with a history of severe arterial hypertension and LVH (IVST and LVIWT, $17 \mathrm{~mm}$ ). Low CI presented after weaning from CPB, and LVOTO was diagnosed with TEE $20 \mathrm{~min}$ after protamine administration. The typical Echocardiographic image of SAM with MR IV and the peak gradient in LVOT of 44 mmHg were present. However, standard therapy (volume loading, metoprolol, and phenylephrine) did not yield a consistent effect, and AV pacing with short (70 ms) delay was started, resulting in full recovery of the intracardiac circulation. Surprisingly, CI remained extremely low: 1.7 before and $1.9 \mathrm{~L}^{*} \mathrm{~min}^{-1 *} \mathrm{~m}^{-2}$ after SAM termination. Restrictive type of hemodynamics was observed: PAWP $>20 \mathrm{mmHg}$, restrictive pattern of mitral inflow [20] with giant E-wave, and small A-wave on PW Doppler, as well as IVST and LVIWT $>20 \mathrm{~mm}$, and systolic LV obliteration. Severe LCOS without SAM led to the patient's death 8 days postoperatively.

The next case showed a similar situation with opposite outcome. A 79-year-old woman underwent on-pump CABG surgery (three grafts). LVOTO was diagnosed by TEE, and she was successfully treated with metoprolol and volume loading $1.5 \mathrm{~h}$ after the patient's arrival in the ICU. However, the significant LCOS remained in the background of LVH (LVIWT and IVST $>20 \mathrm{~mm}$, LV systolic obliteration). The right atrial pressure (RAP), PAWP, hear rate (HR), CI, and AP were $21 \mathrm{mmHg}, 16 \mathrm{mmHg}, 64 \mathrm{~min}^{-1}$, 1.8 $\mathrm{L}^{*} \mathrm{~min}^{-1 *} \mathrm{~m}^{-2}$, and 125/64 $\mathrm{mmHg}$, respectively. Despite obvious risk of inotropes, dobutamine (up to 8 $\mu \mathrm{g}^{*} \mathrm{~kg}^{-1 *} \mathrm{~min}^{-1}$ ) was applied to restore right ventricular function. Thus, we observed a $\mathrm{CI}$ increase to 2.6 


\section{Anaesthesia \& Critical Care Medicine Journal}

$\mathrm{L}^{*} \mathrm{~min}^{-1 *} \mathrm{~m}^{-2}$ with HR of $78 \mathrm{~min}^{-1}$, RAP of 12 , and PAWP of $18 \mathrm{mmHg}$. The restrictive pattern of the mitral inflow was detected before and after the start of dobutamine infusion. No SAM recurrence was observed on TEE. The patient was extubated $12 \mathrm{~h}$ postoperatively, dobutamine infusion was terminated, and the patient was discharged from the ICU two days postoperatively. Thus, all types of responses to therapeutic measures are summarized in the Table 2 and discussed below Table 2 .

\begin{tabular}{|c|c|c|c|c|}
\hline $\begin{array}{c}\text { Response to } \\
\text { therapy }\end{array}$ & $\mathbf{N}$ & $\begin{array}{c}\text { Type of } \\
\text { surgery }\end{array}$ & Died & $\begin{array}{c}\text { Common terms } \\
{[\mathbf{4 , 5 ]}}\end{array}$ \\
\hline Full recovery & 10 & $\begin{array}{c}\text { CABG }-9, \\
\text { AVR }-1\end{array}$ & 0 & $\begin{array}{c}\text { Easy-to-revert or } \\
\text { difficult-to-revert }\end{array}$ \\
\hline Resistant SAM & 1 & CABG & 1 & Persistent SAM \\
\hline $\begin{array}{c}\text { SAM termination } \\
\text { with persistent } \\
\text { LCOS }\end{array}$ & 2 & CABG & 1 & Not described \\
\hline
\end{tabular}

Table 2: Observed responses to therapy of acute LVOTO.

\section{Discussion}

Previously, cases of SAM due to acute myocardial infarction (AMI), during urgent CABG, and after AVR were presented in a few case reports $[8,9,21]$. The most significant studies with systemic analysis of acute SAM in cardiac surgery performed recently have addressed MVR. Crescenzi, et al. [4] and Landoni, et al. [5] presented the most detailed analysis of SAM after MVR and offered the following management steps: step 1 - expanding intravascular volume and discontinuing any inotropic drug; and step 2 - increasing the after load through manual compression of the ascending aorta while administering an intravenous bolus of esmolol. The authors described three types of response to the therapy: "easy-to-revert" (step 1 was effective), "difficult-torevert" (step 2 was effective), and "persistent" (repeated surgical procedure was required). Accordingly, we analyzed some specific mechanisms of LVOTO, with IVS thickening (bulging Subaortic septum) [1,22] manifesting after aorta cross-clamping as the most important. Significant IVST increases can be hypothetically associated with reperfusion myocardial edema in basal IVS, but the specific mechanism of this reperfusion injury remains unclear.

Our single-center study was unable to observe the multitudinous group of patients with this uncommon hemodynamic disturbance. Despite this limitation, the three variants of response to therapy can be described as follows: (1) intracardiac and systemic hemodynamic recovery (most cases) - easy-to-revert or difficult-torevert based on Crescenzi et al. and Landoni, et al. [4,5];
(2) full resistance to therapy with sustainable SAM and LCOS persistence (so-called "persistent SAM" [4,5]); (3) termination of SAM, but paradoxical LCOS persistence due to extremely severe concentric LVH (Table 2). Thus, LVOTO is not only the obvious direct cause of the severe hemodynamic disorders, and it could also be a marker of hard concentric LVH with extremely severe diastolic dysfunction. The persistence of SAM is the most significant, but not always the single pathogenic mechanism of circulatory insufficiency. Actually, in the abovementioned patient, there was a lowest peak gradient in LVOT. The role of restrictive LV remodeling with reduced diastolic compliance is not less important. Selecting a treatment strategy for such cases can be difficult. Traditionally, inotropic therapy has been considered as "a crime" in patients with LVH and SAM. However, we have paradoxically experienced the effective application of inotropes in this case. In our opinion, the useful effects of inotropic agents are an improvement of the right ventricular pump function, leading to additional volume loading of restrictive LV, and HR increase in cases with rigid small stroke volume. Previously, van der Maaten, et al. [23] expressed highly original views on the opportunity for medical improvement of the left atrial pump function. Its rise with inotropic agents immediately after AS correction, along with the evidence that enoximone does not degrade the hypertrophied myocardium compliance, was reported. The necessary condition for providing such extraordinary therapy in patients with severe LVH is careful Echocardiographic control.

In our experience, LVOTO detection was almost always unexpected. This complication was predicted in only 1 of 13 cases studied. This study is an additional argument in favor of routine TEE monitoring in cardiac and major vascular surgeries [24]. We also do not oppose the use of PAC, although it is debatable [25]. The frequency of the use of Swan-Ganz catheter is not decreasing, and the mortality in patients undergoing PAC application during cardiac procedures tended to be lower [26]. However, only echocardiography can identify the real mechanism of circulatory failure. We agree with the approach presented earlier $[4,5]$, demonstrating that a standardized treatment algorithm is essential for rapid detection of cases of persistent SAM. The surgical treatment of this complication after non-mitral valve cardiac procedures has not been commonly implemented. In 2015, Lee et al. reported the successful use of alcohol and albuminglutaraldehyde (BioGlue) for septal ablation to percutaneously treat LVOTO immediately after aortic and mitral valve replacement [6]. Topical case reports of the 


\section{Anaesthesia \& Critical Care Medicine Journal}

MitraClip application for SAM removal were published [27-29].

In conclusion, in the practice of cardiovascular anesthesia, LVOTO, which is not associated with MVR, is an uncommon but dangerous complication. Practitioner vigilance and Echocardiographic monitoring are needed for early SAM detection and effective management. The development of LVOTO during or after non-mitral valve cardiovascular procedures can be a marker of the extremely hard concentric LVH with diastolic restriction as a cause of the low flow status.

\section{References}

1. Ibrahim M, Rao C, Ashrafian H, Chaudhry U, Darzi A, et al. (2012) Modern management of systolic anterior motion of the mitral valve. Eur J Cardiothorac Surg 41(6): 1260-1270.

2. Wigle ED, Rakowski H, Kimball BP, Williams WG (1995) Hypertrophic cardiomyopathy. Clinical spectrum and treatment. Circulation 92(7): 16801692.

3. Termini BA, Jackson PA, Williams CD (1977) Systolic anterior motion of the mitral valve following annuloplasty. Vasc Surg 11(2): 55-60.

4. Crescenzi G, Landoni G, Zangrillo A, Guarracino F, Rosica C, et al. (2009) Management and decisionmaking strategy for systolic anterior motion after mitral valve repair. J Thorac Cardiovasc Surg 137(2): 320-325.

5. Landoni G, Crescenzi G, Zangrillo A, Nicolotti D, Bignami E, et al. (2011) Validation of a decisionmaking strategy for systolic anterior motion following mitral valve repair. Ann Card Anaesth 14(2): 85-90.

6. Lee JZ, Tey KR, Mizyed A, Hennemeyer CT, Janardhanan R, et al. (2015) Mitral valve replacement complicated by iatrogenic left ventricular outflow obstruction and paravalvular leak: case report and review of literature. BMC Cardiovasc Disord 15: 119.

7. Varghese R, Itagaki S, Anyanwu AC, Trigo P, Fischer G, et al. (2014) Predicting systolic anterior motion after mitral valve reconstruction: using intraoperative transoesophageal echocardiography to identify those at greatest risk. Eur J Cardiothorac Surg 45(1): 132137.
8. Xu J, Wen J, Shu L, Liu C, Zhang J, et al. (2007) Mechanism and correlated factors of SAM phenomenon after aortic valve replacement. J Huazhong Univ Sci Technolog Med Sci 27(1): 72-74.

9. Matyal R, Warraich HJ, Karthik S, Panzica P, Shahul S, et al. (2011) Anterior myocardial infarction with dynamic left ventricular outflow tract obstruction. Ann Thorac Surg 91(3): 39-40.

10. Aniskevich S, Shine TS, Feinglass NG, Stapelfeldt WHJ (2007) Dynamic left ventricular outflow tract obstruction during liver transplantation: the role of transesophageal echocardiography. Cardiothorac Vasc Anesth 21(4): 577-580.

11. Luckner G, Margreiter J, Jochberger S, Mayr V, Luger $\mathrm{T}$, et al. (2005) Systolic anterior motion of the mitral valve with left ventricular outflow tract obstruction: Three cases of acute perioperative hypotension in noncardiac surgery. Anesth Analg 100(6): 1594-1598.

12. Chockalingam A, Dorairajan S, Bhalla M, Dellsperger KC (2009) Unexplained hypotension: The spectrum of dynamic left ventricular outflow tract obstruction in critical care settings. Crit Care Med 37(2): 729-734.

13. Reddy S, Ueda K (2004) unexpected refractory intraoperative hypotension during non-cardiac surgery: Diagnosis and management guided by transoesophageal echocardiography. Indian $\mathrm{J}$ Anaesth 58(1): 51-54.

14. Cavallaro F, Marano C, Sandroni C, Dell'anna A (2010) Systolic anterior motion causing hemodynamic instability and pulmonary edema during bleeding. Minerva Anestesiol 76(8): 653-656.

15. Paonessa JR, Brennan T, Pimentel M, Steinhaus D, Feng M, et al. (2015) Hyper dynamic left ventricular ejection fraction in the intensive care unit. Critical Care 19: 288-295.

16. Maslow AD, Regan MM, Haering JM, Johnson RG, Levine RA (1999) Echocardiographic predictors of left ventricular outflow tract obstruction and systolic anterior motion of the mitral valve after mitral valve reconstruction for myxomatous valve disease. J Am Coll Cardiol 34(7): 2096-2104.

17. Myers PO, Khalpey Z, Maloney AM, Brinster DR, D'Ambra MN, et al. (2013) Edge-to-edge repair for prevention and treatment of mitral valve systolic anterior motion. J Thorac Cardiovasc Surg 146(4): 836-840. 


\section{Anaesthesia \& Critical Care Medicine Journal}

18. Timek TA, Nielsen SL, Green GR, Dagum P, Bolger AF, et al. (2001) Influence of anterior mitral leaflet second-order chordae on leaflet dynamics and valve competence. Ann Thorac Surg 72: 535-540.

19. Elsayes A, Trzcinka A (2018) SAM: An unwelcome guest in the OR. J Cardiothorac Vasc Anesth 32(4): 1828.

20. Nishimura RA, Tajik J (1997) Evaluation of diastolic filling of left ventricle in health and disease: Doppler echocardiography is the clinician's Rosetta Stone. J Am Coll Cardiol 30(1): 8-18.

21. Hrovatin E, Piazza R, Pavan D, Mimo R, Macor F, et al. (2002) Dynamic left ventricular outflow tract obstruction in the setting of acute anterior myocardial infarction: a serious and potentially fatal complication? Echocardiography 19(6): 449-455.

22. Canepa M, Malti O, David M, AlGhatrif M, Strait JB, et al. (2014) Prevalence, Clinical Correlates, and Functional Impact of Subaortic Ventricular Septal Bulge (from the Baltimore Longitudinal Study of Aging). Am J Cardiol 114(5): 796-802.

23. Van der Maaten J, De Vries A, Rietman GW, Gallandat Huet RC, De Hert SG (2007) Effects of preemptive enoximone on left ventricular diastolic function after valve replacement for aortic stenosis. J Cardiothorac Vasc Anesth 21(3): 357-366.
24. Geisen M, Spray D, Fletcher SN (2014) Echocardiography-based hemodynamic management in the cardiac surgical intensive care unit. J Cardiothorac Vasc Anesth 28(3): 733-744.

25. Ranucci M (2006) which cardiac surgical patients can benefit from placement of a pulmonary artery catheter? Critical Care 10 (3): S6.

26. Brovman EY, Gabriel RA, Dutton RP, Urman RD (2016) Pulmonary artery catheter use during cardiac surgery in the United States, 2010 to 2014. J Cardiothorac Vasc Anesth 30(3): 579-584.

27. Schäfer U, Frerker C, Thielsen T, Schewel D, Bader R, et al. (2015) Targeting systolic anterior motion and left ventricular outflow tract obstruction in hypertrophic obstructed cardiomyopathy with a MitraClip. EuroIntervention 11 (8): 942-947.

28. Schäfer U, Kreidel F, Frerker C, Schewel D, Bader R, et al. (2014) MitraClip implantation as a new treatment strategy against systolic anterior motion-induced outflow tract obstruction in hypertrophic obstructive cardiomyopathy. Heart Lung Circ 23(5): e131-e135.

29. Agricola E, Taramasso M, Marini C, Montorfano M, Godino C, et al. (2014) First-in-Man MitraClip Implantation to Treat Late Postoperative Systolic Anterior Motion. Circulation 7(6): 860-862. 